Published in final edited form as:

Gynecol Oncol. 2012 December ; 127(3): 538-543. doi:10.1016/j.ygyno.2012.08.020.

\title{
A Phase II Evaluation of Aflibercept in the Treatment of Recurrent or Persistent Endometrial Cancer: a Gynecologic Oncology Group study
}

\author{
Robert L. Coleman, MD ${ }^{1}$, Michael W. Sill, $\mathrm{PhD}^{2}$, Heather A. Lankes, PhD, $\mathrm{MPH}^{2}$, Amanda \\ Nickles Fader, MD ${ }^{3}$, Neil J. Finkler, MD ${ }^{4}$, James S. Hoffman, MD ${ }^{5}$, Peter G. Rose, MD ${ }^{6}$, \\ Gregory P. Sutton, MD7, Charles W. Drescher, MD ${ }^{8}$, D. Scott McMeekin, MD ${ }^{9}$, Wei Hu, MD 1 , \\ Michael Deavers, MD ${ }^{10}$, Andrew K. Godwin, MD $^{12}$, R. Katherine Alpaugh, PhD ${ }^{13}$, and Anil K. \\ Sood, MD ${ }^{11}$ \\ ${ }^{1}$ Dept. of Gynecologic Oncology \& Reproductive Medicine, University of Texas, M.D. Anderson \\ Cancer Center, Houston, TX 77030
}

${ }^{2}$ Gynecologic Oncology Group Statistical \& Data Center; Roswell Park Cancer Institute; Buffalo, NY 14263

${ }^{3}$ Gynecologic Oncology/Clinical Trials Dept; Walter Reed National Military Medical Center Bethesda; Bethesda, MD 20889

${ }^{4}$ Dept. of Obstetrics \& Gynecology, Division of Gynecologic Oncology; Florida Hospital Cancer Institute; Orlando, FL 32804

${ }^{5}$ Dept. of Obstetrics \& Gynecology, Division of Gynecologic Oncology; The Hospital of Central Connecticut; New Britain, CT 06050

${ }^{6}$ Dept. of Obstetrics \& Gynecology, Division of Gynecologic Oncology; Cleveland Clinic Foundation; Cleveland, $\mathrm{OH} 44195$

${ }^{7}$ Dept. of Obstetrics \& Gynecology, Division of Gynecologic Oncology; Saint Vincent's Hospital; Indianapolis, IN 46260

${ }^{8}$ Pacific Gynecologic Specialists, PD; Seattle, WA 98104

${ }^{9}$ Dept. of Obstetrics \& Gynecology, Division of Gynecologic Oncology; University of Oklahoma; Oklahoma City, OK 73190

${ }^{10}$ Dept. of Pathology; University of Texas, M.D. Anderson Cancer Center; Houston, TX 77030

${ }^{11}$ Departments of Gynecologic Oncology \& Reproductive Medicine and Cancer Biology; Center for RNA Interference and Non-Coding RNA, University of Texas, M.D. Anderson Cancer Center; Houston, TX 77030

(C) 2012 Elsevier Inc. All rights reserved.

Corresponding Author: Robert L. Coleman, MD, Professor \& Vice Chair, Clinical Research, Ann Rife Cox Professor in Gynecology, Department of Gynecologic Oncology, University of Texas, M. D. Anderson Cancer Center, 1155 Herman Pressler Dr., CPB 6.3271, Houston, TX 77030, Phone: 713-745-3357, Fax: 713-792-7586, rcoleman@mdanderson.org.

This trial was registered at clinicaltrials.gov (NCT00462826)

\section{CONFLICT OF INTEREST}

Dr. Robert Coleman received grant funding from Sanofi, who has entered into a business relationship with Regeneron for this compound (Aflibercept). All other co-authors have no conflicts of interest to declare.

Publisher's Disclaimer: This is a PDF file of an unedited manuscript that has been accepted for publication. As a service to our customers we are providing this early version of the manuscript. The manuscript will undergo copyediting, typesetting, and review of the resulting proof before it is published in its final citable form. Please note that during the production process errors may be discovered which could affect the content, and all legal disclaimers that apply to the journal pertain. 


\author{
12Dept. of Pathology; The University of Kansas Medical Center; Kansas City, KS 66160 \\ ${ }^{13}$ Protocol Support Laboratory; Fox Chase Cancer Center; Philadelphia, PA 19111
}

\title{
Abstract
}

Purpose-Aflibercept targets vascular endothelial growth factor and placental growth factor. We evaluated activity and toxicity of aflibercept in recurrent/persistent endometrial cancer patients. Biomarkers and association with clinical characteristics and outcome were explored.

Methods-Eligible patients had measurable disease; $1-2$ prior cytotoxic regimens; performance status 0-2. Aflibercept $4 \mathrm{mg} / \mathrm{kg}$ IV q14 days (28-day cycles) was administered until disease progression or prohibitive toxicity. Primary endpoints were the proportion of patients with progression-free survival at six months (PFS6) and tumor response rate. A flexible two-stage group sequential design to detect $20 \%$ increases in the proportion of patients responding or enduring PFS6 with $90 \%$ power $(a=10 \%)$ was employed.

Results-Forty-nine patients were enrolled; five were excluded: wrong primary (2), second primary (1), wrong cell type (1); never treated (1). Median age was 64 (range 48-83). Eighteen patients (41\%) had two prior regimens; $61 \%(27)$ had prior radiation. The PFS6 rate was $41 \%$; three patients 7\%, 90\% CI:2-17) had partial response. Of note, 10 patients (23\%) met the PFS6 endpoint without starting a subsequent therapy; the remaining eight patients discontinued therapy for toxicity and started another therapy before six months elapsed. Median PFS and overall survival were 2.9 months and 14.6 months, respectively. Significant grade 3/4 toxicities were: cardiovascular $(23 \% / 5 \%)$, constitutional $(7 \% / 0)$, hemorrhage $(2 \% / 5 \%)$, metabolic $(7 \% / 2 \%)$, pain $(18 \% / 0)$. Two treatment-related deaths were recorded: GI perforation (1), arterial rupture (1). FGF1 expression was associated with response.

Conclusions-Aflibercept met pretrial activity parameters, but was associated with significant toxicity at this dose and schedule in this population.

\section{Keywords}

Aflibercept; endometrial cancer; VEGF Trap; Toxicity; Fibroblast growth factor

\section{INTRODUCTION}

Uterine epithelial adenocarcinoma is the most common uterine malignancy and the most common gynecologic malignancy in the US. Approximately $25 \%$ of patients with early stage, and more than $50 \%$ of those with advanced stage cancers, will develop recurrent disease. Excluding local disease recurrences, limited options are available for the majority of patients with distant disease. Clinical activity of single or combination cytotoxic agents has been documented with doxorubicin, cisplatin, ifosfamide, and paclitaxel in this patient population. ${ }^{1-4}$ Given the transient responses with these regimens, there is an important unmet need for endometrial cancer treatment.

Angiogenesis is one of the cardinal processes leading to invasion and metastasis of solid tumors. Accumulating evidence suggests that angiogenesis plays a central role in uterine neoplasms. Studies in preclinical xenograft models suggest targeting angiogenesis could be a fruitful treatment approach, particularly in combination with chemotherapy. ${ }^{5}$ While the prognostic relationship between vascular endothelial growth factor (VEGF) protein expression and endometrial cancer patient survival has been inconsistent, the effects of VEGF in the tumor microenvironment (e.g., on microvessel density) appear to have a clear role in tumor biology. ${ }^{6-13}$ The Gynecologic Oncology Group (GOG) recently conducted a 
single arm phase II trial of bevacizumab, demonstrating clinical activity as monotherapy in women with advanced or recurrent endometrial cancer who have been previously treated. ${ }^{14}$

Aflibercept (VEGF Trap, NSC\#724770) is a novel VEGF ligand binding fusion protein that serves as a "decoy receptor" for VEGF binding at high affinity. ${ }^{15}$ The structural features of this compound also provide strong, picomolar binding affinity for placental growth factor (PIGF). ${ }^{16}$ Aflibercept has undergone investigation in many solid tumors, including lung, ovarian, and colorectal cancers; in the latter, aflibercept added to FOLFIRI (irinotecan, 5fluorouracil, leucovorin) was shown to significantly improve progression-free survival (PFS) and overall survival (OS) in a phase III placebo-controlled trial. ${ }^{17}$

Herein, we describe the clinical activity, observed toxicities, and impact of aflibercept on circulating tumor cells (CTCs), circulating endothelial cells (CECs), and the relationship of tumor fibroblast growth factor (FGF) 1 expression to observed clinical outcome in women with persistent or recurrent endometrial cancer. To our knowledge, this is the first investigation of aflibercept in this population.

\section{PATIENTS AND METHODS}

\section{Patients}

Eligible patients had: histologic confirmation of primary endometrial cancer by central pathology review (GOG Pathology Committee); 1-2 prior cytotoxic regimens; GOG performance status of $0-2$; measurable disease by Response Evaluation Criteria in Solid Tumors (RECIST 1.0) ${ }^{18}$; discontinuation of prior chemotherapy ( 33 weeks) and hormonal therapy ( $\geq 1$ week) before registration; recovery from effects of recent surgery, radiotherapy, or chemotherapy; freedom from active infection requiring antibiotics; adequate hematologic (absolute neutrophil count $\geq 1,500 / \mathrm{mcl}$ and platelets $\geq 100,000 / \mathrm{mcl}$ ), renal (serum creatinine $<1.5$ times the institutional upper limit of normal [ULN] and urine protein-to-creatinine ratio $<1$ ), hepatic (serum bilirubin $\unlhd .5 \mathrm{ULN}$ and AST and alkaline phosphatase $2.5 \mathrm{ULN}$ ), and coagulation laboratory values; normal left ventricular ejection fraction; negative serum pregnancy test; signed approved informed consent in accordance with federal, state, and local requirements; and authorization permitting release of personal health information.

Ineligibility criteria included prior treatment with aflibercept or VEGF-targeted therapy; prior treatment with any non-cytotoxic (except hormonal) therapy; other malignancies (except non-melanomatous skin cancer) evident within five years; non-healing wound; abdominal fistula, gastrointestinal (GI) perforation, or intra-abdominal abscess within 28 days; active bleeding or pathologic condition that carries high risk of bleeding; known metastatic central nervous system (CNS) disease; clinically significant cardiovascular disease; or major surgical procedure within 28 days.

\section{Treatment}

Enrolled patients were to receive aflibercept $4 \mathrm{mg} / \mathrm{kg}$ intravenously every 14 days (one cycle equaled two infusions or 28 days), with no dose modification except for changes in body weight of $\geq 10 \%$. Treatment was planned until disease progression or adverse events prohibited further therapy. Toxicity was monitored before each treatment cycle, with adverse events defined and graded according to Common Terminology Criteria for Adverse Events (version 3). Aflibercept was held for grade 3 non-hematologic toxicity for up to two weeks (in most circumstances; up to four weeks in special circumstances). 'Special circumstances' included prolonged Grade 2 toxicity for which the investigator felt additional delay may be of merit, time for recovery after minor surgical procedure (e.g. infusion port) or patient-requested or centric delays (e.g. weather, travel). Aflibercept was discontinued for grade 4 allergic reactions; grade 2 (new or worsened) - 4 arterial thrombosis; symptomatic 
grade 4 venous thrombosis; grade 4 hypertension; grade 4 proteinuria (or nephrotic syndrome); grade 4 hemorrhage; GI perforation, GI leak, or fistula; and reversible posterior leukoencephalopathy syndrome. Specific treatment guidelines were implemented for hypertension, venous thrombosis, proteinuria, and hemorrhage.

\section{Evaluation Criteria}

Activity of aflibercept was assessed according to RECIST (version 1.0) by palpation before each cycle or by computed tomography or magnetic resonance imaging at baseline, every other cycle for the first six months, and every three months thereafter until documentation of disease progression or as clinically indicated.

Circulating Tumor Cells and Circulating Endothelial Cells-CTCs and CECs were enriched using the Celltracks ${ }^{\mathrm{TM}}$ Autoprep system (Veridex, LLC.) from blood drawn prior to cycles one, two and three of treatment. Cell-specific isolation was achieved using antibodyconjugated ferrofluid. EpCAM-ferrofluid was used to recover CTCs; CD34-ferrofluid to isolate CECs. The Celltracks ${ }^{\mathrm{TM}}$ Analyzer II was used for image analysis. CTCs were defined as CD45-, DAPI+, and anti-cytokeratin+. CTC apoptosis was assessed using the M30 monoclonal antibody. CECs were defined as CD146+, CD34+, DAPI+ and CD45-, and phenotyped using anti-VEGFR2.

Tumor Immunohistochemistry Analysis-Formalin-fixed, paraffin-embedded (FFPE) tumor was used to determine the expression of IL-8, VEGF, VEGF receptor (VEGFR) 2, FGF-1, FGF-2, and microfibril associated glycoprotein (MAGP) 2. Staining details are included in the Supplementary Methods. Immunohistochemistry stains were reviewed by a pathologist (MD). Expression was scored as intensity (1=no/focal, $2=$ light, $3=$ medium, $4=$ dark staining), ordinal ranking of percent positive cells $(0=$ none, $1=1-25 \%, 2=26-50 \%$, $3=51-75 \%, 4=76-100 \%$ ), and an overall score calculated as the intensity plus ordinal ranking of percent positive cells. ${ }^{19}$

\section{Statistical Methods}

The primary objective evaluated the efficacy of aflibercept using a composite endpoint of objective tumor response and the frequency of patients with PFS for at least six months. The null hypothesis relating to uninteresting levels of activity was determined from analysis of a historical data set based on a similar population of patients where the levels of activity were believed to be inactive to modestly active (Table 1, Aghajanian et al). ${ }^{14}$ The null hypothesis jointly specified the probability of a patient experiencing a tumor response to be $10 \%$ or less and the probability of a patient being progression free for at least six months to be $15 \%$ or less. Clinically significant differences were $20 \%$ increases in the probability of either event (i.e., probability of $30 \%$ or $35 \%$, respectively). Using a method by Sill and Yothers,${ }^{20}$ a twostage design was used with a goal of limiting patient exposure to inactive agents, while restricting the probabilities of type I and type II errors to approximately $10 \%$. The targeted accrual for the first stage was 21 patients but allowed to deviate for administrative purposes. If 19 patients were accrued, the critical values for the number of patients with responses or PFS at six months were two and three, respectively. The cumulative targeted accrual for the second stage was 40 , but allowed to deviate. If 44 patients were accrued, the critical values for the number of patients with responses or PFS for at least six months were eight and 10, respectively.

The realized sample sizes limited the probabilities of type I and II errors to no more than $10 \%$ under the null hypothesis. The probability of early termination was $48 \%-59 \%$, depending on the likely range of association between the two variables. 
Biomarkers were screened for associations with demographic characteristics and clinical outcomes in an exploratory fashion to yield hypotheses for further testing. Characteristics of interest included age, performance status, prior treatment, cell grade, response, PFS until subsequent therapy at six months, and the hazards of progression, death, or treatment failure. Treatment failure was defined as the time when a patient started a subsequent therapy after going off of protocol therapy for reasons unrelated to disease progression; patient's PFS status at six months was classified negatively if the patient had subsequent therapy before six months of study entry. Where appropriate, biomarkers were dichotomized into high versus low values using the observed median. Some characteristics were examined as ordinal categorical variables (e.g. response with progressive disease < stable disease < response). Associations were examined with correlations (e.g. Spearman rank correlation coefficient), exact Chi-square tests, Jonckheere-Terpstra test, and Cox proportional hazards model. ${ }^{21}$ Changes in levels of CEC and \%VEGFR2 were calculated for each patient and examined through the median change by utilizing distribution free methods. ${ }^{22}$ If a p-value was $\mathbf{5 \%}$, an association was designated as suggestive. Given the small sample sizes, a lack of a suggestive association should not be interpreted as definitive.

\section{RESULTS}

\section{Patient Characteristics}

Forty-nine patients were enrolled from November 2007 through July 2009; four were excluded - wrong primary (2), second primary (1), wrong cell type (1). One additional patient was never treated, leaving 44 evaluable patients for toxicity and response. Table 1 presents patient characteristics. Of note, more than $40 \%$ of patients received two prior regimens of therapy; $61 \%$ received prior radiation therapy. Thirty-nine percent of patients received three or more cycles of study therapy; $18 \%$ received seven or more.

\section{Adverse Events}

Table 2 lists hematological and non-hematological toxicities. Two fatal (grade 5) events were observed with attributions listed as at least possibly related to study agent. One patient suffered a GI perforation after one cycle of therapy. She was not offered corrective surgery due to disease status and illness. The second was a 69-year-old patient who died after 19 cycles from a ruptured artery. There were four other deaths on study attributed to treatment and disease progression. The most common grade 3 or 4 toxicity observed was hypertension ( $\mathrm{n}=10$ and 2, respectively. Although only two patients suffered grade 2 neurosensory toxicity, five had grade 3 or 4 neurological effects, including two with reversible posterior leukoencephalopathy syndrome (RPLS). Each of these two patients had a severe hypertension event before presenting with symptoms of confusion, disorientation, and seizure. One patient had an antecedent history of paraplegia from a motor vehicle accident. Other serious events included life threatening CNS hemorrhage (1), hematoma (1), and hypoxia (1), as well as severe hemorrhage in the lung (1), dyspnea (3), and pain (8). In all, 14 evaluable patients (32\%) were removed from study because of toxicity with one refusing additional therapy (Table 3).

\section{Clinical Activity}

There were no complete responders; three partial responses were seen for an overall response rate (RR) 7\% (90\% CI, 2-17\%). Two of these patients had endometrioid adenocarcinoma and one had clear cell; each of these patients had diffuse metastatic disease. Stable disease was observed in 14 patients (32\%) and 10 are alive with (7) or without (3) disease progression. The most frequent cause of mortality was disease $79 \%(27 / 34)$. Eighteen patients were progression-free at six months (41\%; 90\% CI: 28\%-54\%). Only 10 (23\%) were progression-free for six months without starting a subsequent therapy; eight of 
the 18 patients classified as PFS at six months went off therapy for toxicity and started another therapy before six months elapsed from entering the current study. No differences in response or non-progression were observed by histology type. PFS and OS Kaplan Meier survival curves are presented in Figure 1. The median PFS was 2.9 months (90\% CI: $2.1-$ 6.21 mos); median OS, 14.5 months (90\% CI: 9.86-20.44 months).

CTC and CEC Evaluation-Thirty-six of the 44 patients who participated in correlative testing had a baseline sample. Of those $36,10 \mathrm{had} \geq 1$ CTC at baseline (range 1-49). M30, an apoptosis marker, could not be evaluated due to the lack of CTCs. Table 4 shows the median number of CECs and percent VEGFR2+ CECs at each time point. The median change in CECs from pre-cycle 1 to pre-cycle (course) 2 was 7 (95\% CI: -22-26), which was not suggestive. Pre-treatment number of CECs was not associated with response nor patient PFS status at six months. The median change in the percent of VEGFR2+ cells was -0.068 (95\% CI: $-0.111-0.030$ ) from pre-cycle 1 to pre-cycle 2 , which was not suggestive. Pre-treatment percent of VEGFR2+ CECs was not associated with clinical characteristics, response, nor PFS status at six months.

Immunohistochemistry-Expression of VEGF, VEGFR2, IL8, FGF1, FGF2 and MAPG2 in FFPE primary tumor was examined by immunohistochemistry. Table 5 shows the median intensity for each biomarker. Expression of FGF1 (intensity, ordinal ranking of percent positive cells, and overall score) was suggestively associated with GOG performance status and response ( $\mathrm{r}=-0.61$ and 0.55 , respectively). Higher FGF1 expression was associated with good performance status and response. There was also a trend of positive correlation between the number of CECs and FGF1 expression in tumor, as detected by Spearman's correlation $(\mathrm{r}=0.51) .^{20}$

\section{DISCUSSION}

This phase II study suggests aflibercept is associated with a beneficial effect on disease progression, with $41 \%$ of recurrent/persistent endometrial cancer patients experiencing PFS of six months or longer. In light of the available options for patients with recurrent, previously treated endometrial cancer, this clinical activity is noteworthy. However, while this observation met our predefined primary endpoints for clinical interest and future examination, both the associated on-treatment toxicity observations and the delay in disease progression calculation must be carefully considered prior to future development. First, the population in this clinical cohort was similar to those entering other GOG phase II trials in this protocol queue, which was primarily designed to evaluate the impact of biologicallybased therapy on response and disease progression. However, the regimen at this dose and infusion schedule appeared to be more toxic than other agents evaluated in this queue. For instance, treatment discontinuation in the absence of disease progression occurred in $32 \%$ of patients on this trial; by way of comparison, just $6 \%$ of women receiving bevacizumab in a previous GOG phase II study in recurrent/persistent endometrial cancer patients (GOG 0229E) discontinued therapy prior to progression. ${ }^{14}$

Second, there were two deaths attributed to adverse events considered at least possibly related to aflibercept (GI perforation and arterial rupture) and (four other deaths) considered related to disease progression and therapy. Third, while traditional adverse events associated with anti-VEGF ligand therapy such as hypertension, proteinuria, and neurological symptoms were observed with aflibercept, grade 3-4 events were higher than expected and included two patients with RPLS.

Finally, the high rate of treatment discontinuation observed in this trial may have inappropriately inflated our estimation of clinical benefit represented by non-progression at 
six months. This endpoint is used to quantify the clinical benefit patients with otherwise stable disease, based on RECIST parameters, may achieve on therapy. Under normal circumstances, patients demonstrating progression on therapy would be considered as treatment failures, and those with response or durable non-progressive disease would be considered as gaining benefit from therapy. However, patients discontinuing therapy in the absence of progression are considered evaluable until progression is documented, regardless of subsequent therapy (if any). When treatment discontinuation is a small fraction of the total, the influence of censoring is minimal; however, as may have been observed in the current trial, a large fraction could highlight the merit of post-progression therapy. If these patients were reclassified as treatment failures, our estimation of non-progression at six months would be $23 \%$.

In this study, we also sought to enumerate and characterize CTCs and CECs from blood drawn before and during treatment with aflibercept. This was done to explore whether any one or combination of circulating cells can serve as surrogates of drug effects and mechanisms of action at tumor sites. Ten of 36 patients (28\%) with baseline samples had one or more CTC. There was a suggested association with higher numbers of CEC at baseline, which possibly reflects high vascular turnover and remodeling, and vascular injury and damage. The median number of CECs increased from pre-cycle 1 to pre-cycle 2; however, this result was not suggestive of an overall or systematic increase over time. Whether CEC level can be predictive to anti-VEGF treatment is still controversial. Conflicting results of the clinical predictive value of CEC level have been reported in cancer patients treated with anti-VEGF drugs, including aflibercept ${ }^{23}$ mainly due to different CEC phenotypes (CD105+CD146+CD45-, CD34+CD146+CD45-CD133-), different clinical situations, timing of collection, and different techniques used for CEC detection. We also found higher FGF1 expression was associated with response and good performance status. Since it is well known that patients with good performance status tend to be more likely to respond, a causal mechanism between these variables is not clearly discerned in this study. There was a trend involving Spearman's correlation between performance status and response in this patient population $(\mathrm{r}=-0.29)$; $44 \%$ of patients with increasing disease had poor performance status, $21 \%$ with stable disease had poor performance status, and none of the responders had poor performance status. ${ }^{20}$ Moreover, there was also a trend of positive correlation between the number of CECs and FGF1 expression in tumor. It has been suggested that FGF1 is a potent angiogenic factor for endothelial cell proliferation and promoting angiogenesis. FGF1 is a prognostic marker of advanced-stage ovarian cancer. ${ }^{24,25}$ However, whether FGF1 expression in tumor tissues and/or on CECs can be used as a predictive marker for this treatment will require validation in larger studies.

In summary, aflibercept, at this dose and schedule, fell within the clinical activity range to warrant further investigation but should be done cautiously given the toxicity observed in this phase II trial. Other infusion schedules have been investigated, including the 21-day schedule in ovarian cancer. As a single agent and in combination with chemotherapy, aflibercept has been well tolerated in the 21-day schedule and may represent the best investigative path forward for this agent in women with recurrent or advanced endometrial cancer.

\section{Supplementary Material}

Refer to Web version on PubMed Central for supplementary material. 


\section{Acknowledgments}

This study was supported by National Cancer Institute grants to the Gynecologic Oncology Group Administrative Office (CA 27469) and the Gynecologic Oncology Group Statistical and Data Center (CA 37517). This was also supported, in part, by NIH grant P50 CA098258. The following Gynecologic Oncology Group member institutions participated in this study: Duke University Medical Center, Walter Reed Army Medical Center, Northwestern Memorial Hospital, University of Washington, University of North Carolina School of Medicine, University of New Mexico, The Cleveland Clinic Foundation, Washington University School of Medicine, Columbus Cancer Council, MD Anderson Cancer Center, Fox Chase Cancer Center, University of Oklahoma, Case Western Reserve University, University of Wisconsin, The Hospital of Central Connecticut, and Community Clinical Oncology Program. RLC is supported in part by the Ann Rife Cox Chair in Gynecology.

CellSearch Endothelial kits were supplied by Veridex, LLC (Huntington Valley, PA) as part of a collaborative research agreement with Fox Chase Cancer Center and Veridex. We also acknowledge Regeneron for providing the investigational drug.

The authors would like to thank Sandy Dascomb for data management, Diana Blade for protocol administration, Kim Blaser in publication, and the GOG Tissue Bank for their assistance with the banking and distribution of specimens and Veridex, LLC for providing CelSearch ${ }^{\mathrm{TM}}$ Epithelial Cell research kits, and De-Yu Shen in the Department of Gynecologic Oncology and Reproductive Medicine at MD Anderson Cancer Center for performing immunochemistry staining.

\section{References}

1. Duska LR, Berkowitz R, Matulonis U, et al. A pilot trial of TAC (paclitaxel, doxorubicin, and carboplatin) chemotherapy with filgastrim (r-metHuG-CSF) support followed by radiotherapy in patients with "high-risk" endometrial cancer. Gynecol Oncol. 2005; 96:198-203. [PubMed: 15589601]

2. Thigpen JT, Brady MF, Homesley HD, et al. Phase III trial of doxorubicin with or without cisplatin in advanced endometrial carcinoma: a gynecologic oncology group study. J Clin Oncol. 2004; 22:3902-8. [PubMed: 15459211]

3. Soper JT, Reisinger SA, Ashbury R, et al. Feasibility study of concurrent weekly cisplatin and whole abdominopelvic irradiation followed by doxorubicin/cisplatin chemotherapy for advanced stage endometrial carcinoma: a Gynecologic Oncology Group trial. Gynecol Oncol. 2004; 95:95100. [PubMed: 15385116]

4. Fleming GF, Brunetto VL, Cella D, et al. Phase III trial of doxorubicin plus cisplatin with or without paclitaxel plus filgrastim in advanced endometrial carcinoma: a Gynecologic Oncology Group Study. J Clin Oncol. 2004; 22:2159-66. [PubMed: 15169803]

5. Kamat AA, Merritt WM, Coffey D, et al. Clinical and biological significance of vascular endothelial growth factor in endometrial cancer. Clin Cancer Res. 2007; 13:7487-95. [PubMed: 18094433]

6. Talvensaari-Mattila A, Soini Y, Santala M. VEGF and its receptors (flt-1 and KDR/flk-1) as prognostic indicators in endometrial carcinoma. Tumour Biol. 2005; 26:81-7. [PubMed: 15867479]

7. Fine BA, Valente PT, Feinstein GI, et al. VEGF, flt-1, and KDR/flk-1 as prognostic indicators in endometrial carcinoma. Gynecol Oncol. 2000; 76:33-9. [PubMed: 10620438]

8. Fujiwaki R, Hata K, Iida K, et al. Co-expression of vascular endothelial growth factor and thymidine phosphorylase in endometrial cancer. Acta Obstet Gynecol Scand. 1999; 78:728-34. [PubMed: 10468067]

9. Yokoyama Y, Charnock-Jones DS, Licence D, et al. Expression of vascular endothelial growth factor (VEGF)-D and its receptor, VEGF receptor 3, as a prognostic factor in endometrial carcinoma. Clin Cancer Res. 2003; 9:1361-9. [PubMed: 12684405]

10. Sivridis E, Giatromanolaki A, Anastasiadis P, et al. Angiogenic co-operation of VEGF and stromal cell TP in endometrial carcinomas. J Pathol. 2002; 196:416-22. [PubMed: 11920737]

11. Giatromanolaki A, Sivridis E, Brekken R, et al. The angiogenic "vascular endothelial growth factor/flk-1(KDR) receptor" pathway in patients with endometrial carcinoma: prognostic and therapeutic implications. Cancer. 2001; 92:2569-77. [PubMed: 11745191]

12. Chen CA, Cheng WF, Lee CN, et al. Cytosol vascular endothelial growth factor in endometrial carcinoma: correlation with disease-free survival. Gynecol Oncol. 2001; 80:207-12. [PubMed: $11161861]$ 
13. Hirai M, Nakagawara A, Oosaki T, et al. Expression of vascular endothelial growth factors (VEGF-A/VEGF-1 and VEGF-C/VEGF-2) in postmenopausal uterine endometrial carcinoma. Gynecol Oncol. 2001; 80:181-8. [PubMed: 11161857]

14. Aghajanian C, Sill MW, Darcy KM, et al. Phase II Trial of Bevacizumab in Recurrent or Persistent Endometrial Cancer: A Gynecologic Oncology Group Study. Journal of clinical oncology: official journal of the American Society of Clinical Oncology. 2011; 29:2259-65. [PubMed: 21537039]

15. Holash J, Davis S, Papadopoulos N, et al. VEGF-Trap: a VEGF blocker with potent antitumor effects. Proc Natl Acad Sci U S A. 2002; 99:11393-8. [PubMed: 12177445]

16. Kim ES, Serur A, Huang J, et al. Potent VEGF blockade causes regression of coopted vessels in a model of neuroblastoma. Proc Natl Acad Sci U S A. 2002; 99:11399-404. [PubMed: 12177446]

17. van Cutsem E, Tabernero J, Lakomy R, et al. Intravenous (IV) aflibercept versus placebo in combination with irinotecan/5-FU (FOLFIRI) for second-line treatment of metastatic colorectal cancer (MCRC): Results of a multinational phase III trial (EFC 10262-VELOUR). Annals of Oncology. 2011; 22 v18 (O-0024).

18. Therasse P, Arbuck SG, Eisenhauer EA, et al. New guidelines to evaluate the response to treatment in solid tumors. European Organization for Research and Treatment of Cancer, National Cancer Institute of the United States, National Cancer Institute of Canada.[see comment]. Journal of the National Cancer Institute. 2000; 92:205-16. [PubMed: 10655437]

19. Allred DC, Harvey JM, Berardo M, et al. Prognostic and predictive factors in breast cancer by immunohistochemical analysis. Modern pathology: an official journal of the United States and Canadian Academy of Pathology, Inc. 1998; 11:155-68.

20. Sill, MW.; Yothers, G. Technical Report 06-08. 2008. Buffalo: Department of Biostatistics, University of Buffalo; 2008. A method for utilizing bivariate efficacy outcome measures to screen agents for activity in 2-stage phase II clinical trials.

21. Cox DR. Regression models and life tables. J Royal Statist Soc. 1972; 34:187-220. Series B.

22. Hahn, GJ.; Meeker, WQ. Statistical Intervals: A Guide for Practitioners. New York: John Wiley \& Sons; 1991.

23. Coleman RL, Duska LR, Ramirez PT, et al. Phase 1-2 study of docetaxel plus aflibercept in patients with recurrent ovarian, primary peritoneal, or fallopian tube cancer. The lancet oncology. 2011; 12:1109-17. [PubMed: 21992853]

24. Birrer MJ, Johnson ME, Hao K, et al. Whole genome oligonucleotide-based array comparative genomic hybridization analysis identified fibroblast growth factor 1 as a prognostic marker for advanced-stage serous ovarian adenocarcinomas. Journal of clinical oncology: official journal of the American Society of Clinical Oncology. 2007; 25:2281-7. [PubMed: 17538174]

25. Cao R, Brakenhielm E, Pawliuk R, et al. Angiogenic synergism, vascular stability and improvement of hind-limb ischemia by a combination of PDGF-BB and FGF-2. Nature medicine. 2003; 9:604-13. 


\section{HIGHLIGHTS}

- Anti-angiogenesis targeting in endometrial cancer appears to be a relevant target

- Aflibercept met pretrial efficacy criteria for clinical development interest

- Toxicity at the dose and schedule used in this phase II trial was significant 


\section{Progression-Free Sunival and Survival}

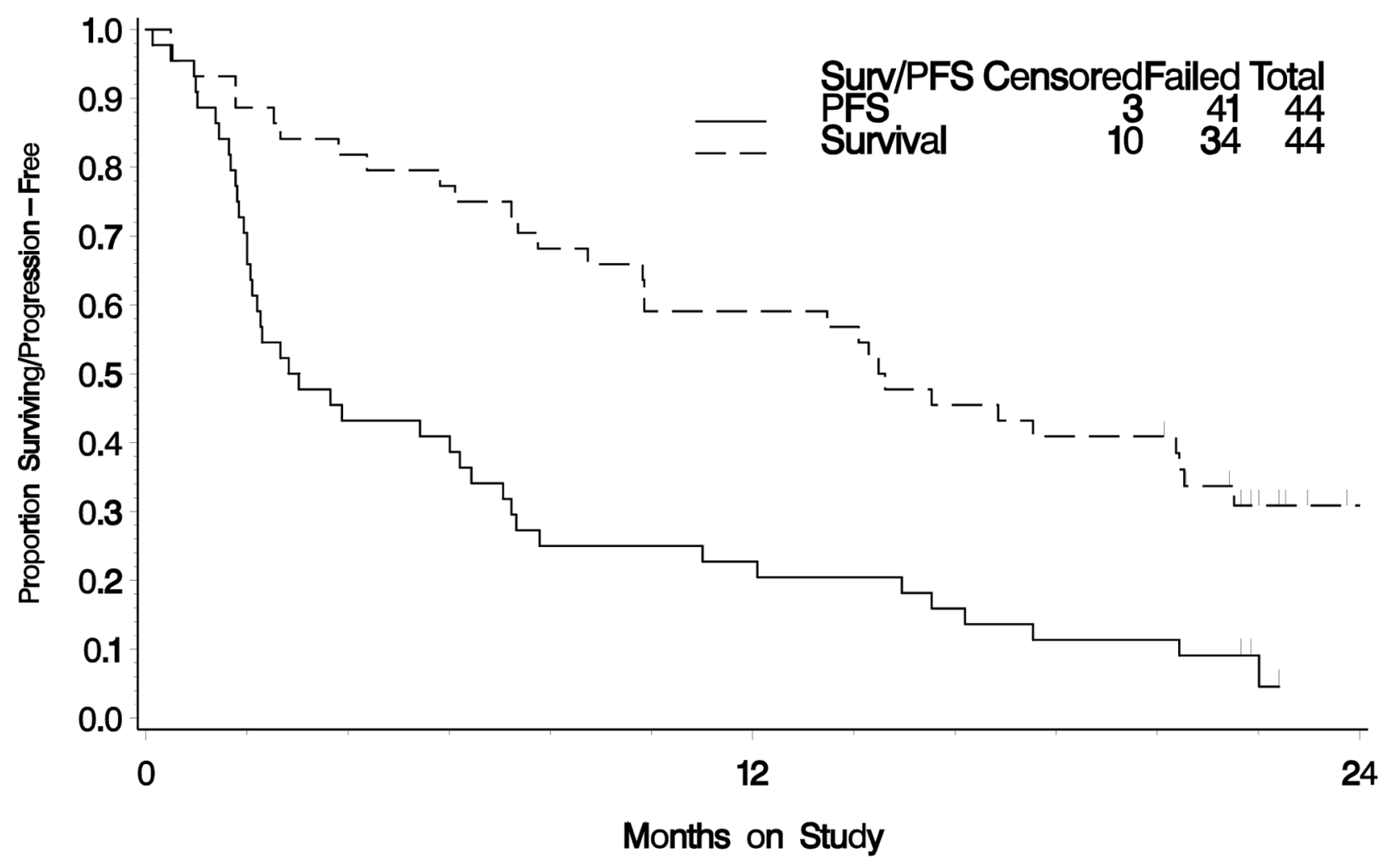

Figure 1.

Kaplan-Meier plots depicting the progression-free and overall survival for the 44 evaluable patients on this trial. 


\section{Table 1}

Patient Characteristics $(\mathrm{N}=44)$

\begin{tabular}{|c|c|c|c|}
\hline Characteristic & Category & No. of Cases & $\%$ of Cases \\
\hline \multirow[t]{5}{*}{ Age } & $40-49$ & 3 & 6.8 \\
\hline & $50-59$ & 13 & 29.5 \\
\hline & $60-69$ & 15 & 34.1 \\
\hline & $70-79$ & 12 & 27.3 \\
\hline & $80-89$ & 1 & 2.3 \\
\hline \multirow[t]{3}{*}{ Race } & Asian & 1 & 2.3 \\
\hline & African American & 4 & 9.1 \\
\hline & White & 39 & 88.6 \\
\hline \multirow[t]{3}{*}{ Performance Status } & 0 & 29 & 65.9 \\
\hline & 1 & 14 & 31.8 \\
\hline & 2 & 1 & 2.3 \\
\hline Site of Disease & Corpus & 44 & 100.0 \\
\hline \multirow[t]{10}{*}{ Cell Type/Grade } & Endometrioid, grade 1 & 2 & 4.5 \\
\hline & Endometrioid, grade 2 & 14 & 31.8 \\
\hline & Endometrioid, grade 3 & 2 & 4.5 \\
\hline & Endometrioid, not graded & 5 & 11.4 \\
\hline & Serous & 11 & 25.0 \\
\hline & Clear Cell & 1 & 2.3 \\
\hline & Mixed Epithelial & 6 & 13.6 \\
\hline & Adenocarcinoma, NOS & 1 & 2.3 \\
\hline & Mucinous & 1 & 2.3 \\
\hline & Other & 1 & 2.3 \\
\hline \multirow[t]{2}{*}{ Prior Chemotherapy } & 1 Prior Regimen & 26 & 59.1 \\
\hline & 2 Prior Regimens & 18 & 40.9 \\
\hline \multirow[t]{2}{*}{ Prior Radiation } & No & 17 & 38.6 \\
\hline & Yes & 27 & 61.4 \\
\hline Prior Immunotherapy & No & 44 & 100.0 \\
\hline \multirow[t]{2}{*}{ Prior Surgery } & No & 2 & 4.5 \\
\hline & Yes & 42 & 95.5 \\
\hline
\end{tabular}

Gynecol Oncol. Author manuscript; available in PMC 2013 December 01. 
Table 3

Adverse events attributed as at least "possibly related" to study drug that lead trial discontinuation *

\begin{tabular}{ll}
\hline Adverse Event & N \\
Hypertensive Crisis or Uncontrolled Hypertension & 4 \\
Cognitive Decline & 3 \\
Proteinuria & 3 \\
Leukoencephalopathy & 2 \\
Dyspnea/Heart failure & 1 \\
Unresolved Elevated Alkaline Phosphatase & 1 \\
Unresolved Hyponatremia & 1 \\
Transient Ischemic Attack & 1 \\
\hline
\end{tabular}

Individuals may have experienced more than one AE resulting in study drug discontinuation 


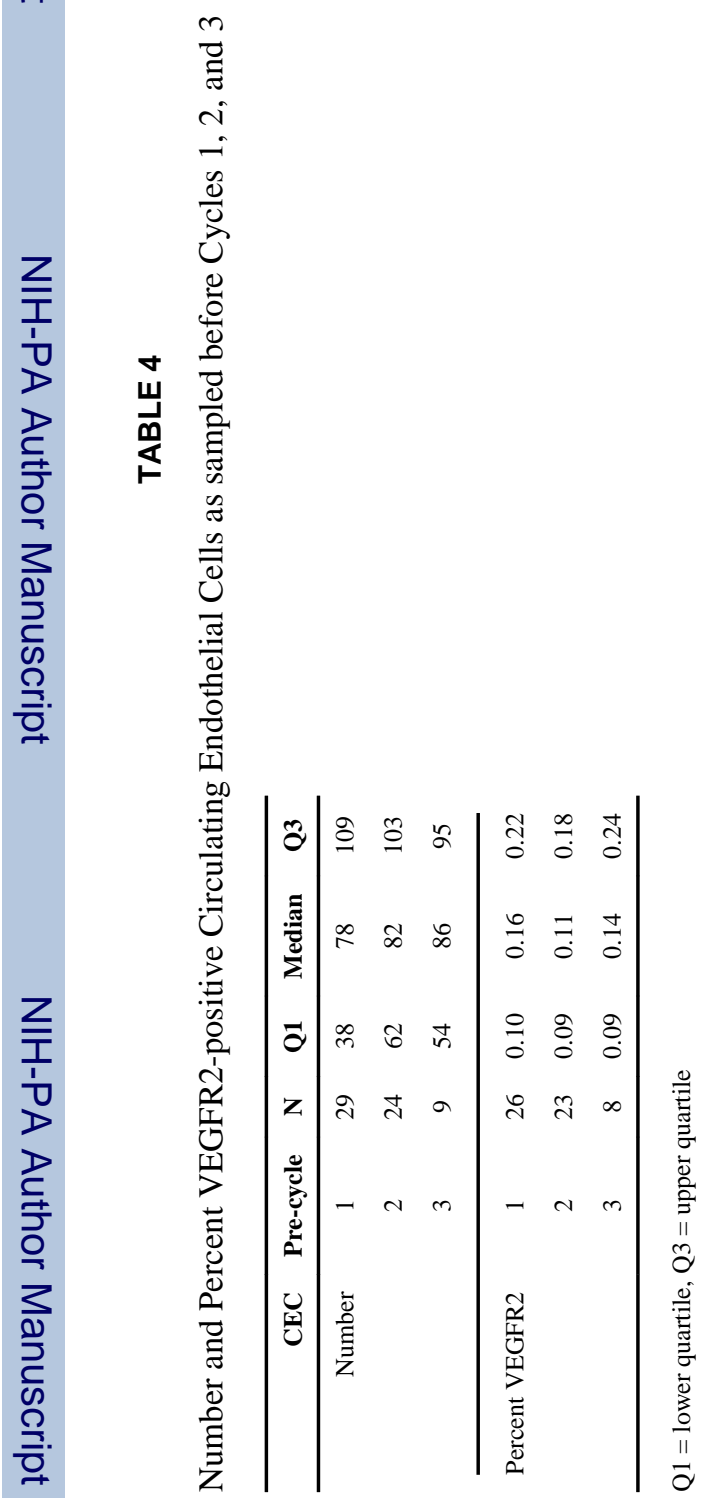




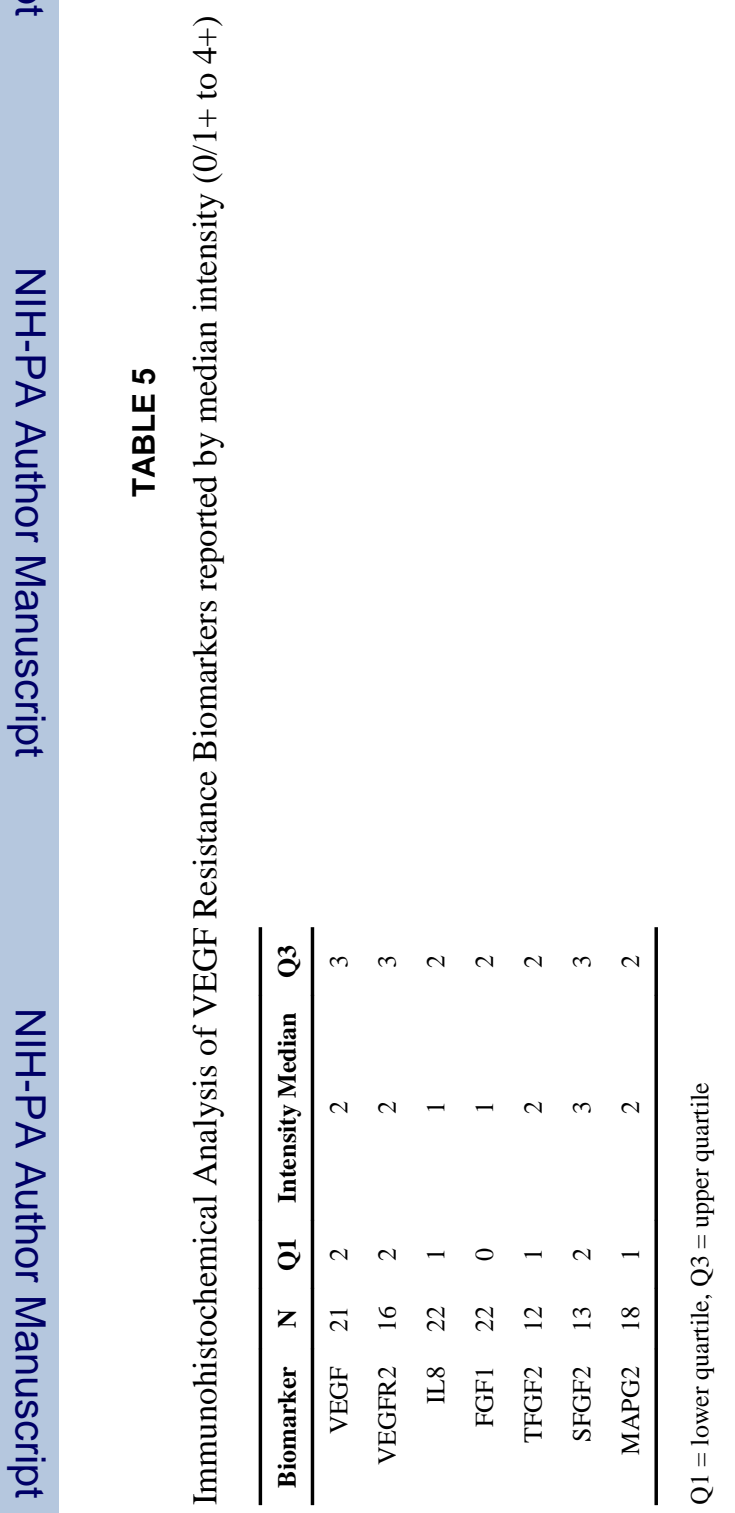

